OPEN ACCESS

Edited by:

Michael Gänzle,

University of Alberta, Canada

Reviewed by:

Maria Elena Martino,

Institut de Génomique Fonctionnelle,

France

Eric Altermann,

AgResearch, New Zealand

*Correspondence:

Sarah Lebeer

sarah.lebeer@uantwerpen.be

Gregor Reid

gregor@uwo.ca

tThese authors are joint senior authors.

Specialty section:

This article was submitted to

Food Microbiology,

a section of the journal

Frontiers in Microbiology

Received: 23 December 2017

Accepted: 25 May 2018

Published: 15 June 2018

Citation:

Petrova MI, Macklaim JM, Wuyts S, Verhoeven T, Vanderleyden J,

Gloor GB, Lebeer S and Reid G (2018) Comparative Genomic and Phenotypic Analysis of the Vaginal Probiotic Lactobacillus

rhamnosus GR-1.

Front. Microbiol. 9:1278.

doi: 10.3389/fmicb.2018.01278

\section{Comparative Genomic and Phenotypic Analysis of the Vaginal Probiotic Lactobacillus rhamnosus GR-1}

\author{
Mariya I. Petrova ${ }^{1,2}$, Jean M. Macklaim ${ }^{3,4}$, Sander Wuyts², Tine Verhoeven', \\ Jos Vanderleyden', Gregory B. Gloor ${ }^{3,4}$, Sarah Lebeer ${ }^{1,2 * t}$ and Gregor Reid ${ }^{3,5,6 * t}$ \\ ${ }^{1}$ Centre of Microbial and Plant Genetics, KU Leuven, Leuven, Belgium, ${ }^{2}$ Department of Bioscience Engineering, University \\ of Antwerp, Antwerp, Belgium, ${ }^{3}$ Canadian Research and Development Centre for Human Microbiome and Probiotics, \\ Lawson Health Research Institute, The University of Western Ontario, London, ON, Canada, ${ }^{4}$ Department of Biochemistry, \\ The University of Western Ontario, London, ON, Canada, ${ }^{5}$ Department of Microbiology and Immunology, The University of \\ Western Ontario, London, ON, Canada, ${ }^{6}$ Department of Surgery, The University of Western Ontario, London, ON, Canada
}

Lactobacillus represents a versatile bacterial genus, which can adapt to a wide variety of ecological niches, including human body sites such as the intestinal and urogenital tract. In this study, the complete genome sequence of the vaginal probiotic Lactobacillus rhamnosus GR-1 was determined and compared to other $L$. rhamnosus strains at genomic and phenotypic level. The strain GR-1 was originally isolated from a female urethra, and was assessed with $L$. rhamnosus GG from a feces sample of a healthy male, and L. rhamnosus LC705 from a dairy product. A key difference is the absence in GR-1 and LC705 of the spaCBA locus required for pili-mediated intestinal epithelial adhesion. In addition, the L. rhamnosus GR-1 genome contains a unique cluster for exopolysaccharide production, which is postulated to synthesize glucose-rich, rhamnose-lacking exopolysaccharide molecules that are different from the galactoserich extracellular polysaccharide of $L$. rhamnosus GG. Compared to $L$. rhamnosus GG, L. rhamnosus GR-1 was also genetically predicted and experimentally shown to better metabolize lactose and maltose, and to better withstand oxidative stress, which is of relevance in the vagina. This study could thus provide a molecular framework for the selection of the optimal probiotic strain for each targeted niche and condition, but further substantiation of niche adaptation mechanisms of lactobacilli is warranted.

Keywords: adaptation, genome, lactobacilli, probiotics, vaginal niche

\section{INTRODUCTION}

The importance of Lactobacillus species in promoting a healthy ecosystem in the human vagina has been well-recognized (Bruce et al., 1973; Petrova et al., 2015). The ability to replenish an aberrant vaginal microbiota with exogenous lactobacilli as probiotics was first reported in 1988 using Lactobacillus rhamnosus GR-1 (Bruce and Reid, 1988). The organism had been isolated from the distal urethra of a healthy female, and shown to counter the growth and adhesion to uroepithelial cells of urinary pathogens, and reduce urinary infection in animas (Chan et al., 1985; Reid et al., 1985). Since then, other attributes of the strain that appear to be important in counteracting 
pathogens have been reported, such as penetration of pathogenic biofilms (McMillan et al., 2011) via, for example, lectin-like proteins (Petrova et al., 2016), and enhancement of host defenses (Kirjavainen et al., 2008). The strain has also been shown to temporarily colonize the human vagina (Gardiner et al., 2002) and intestine in vivo following oral uptake (Reid et al., 2003). The fact that oral application of $L$. rhamnosus GR-1 can result in vaginal colonization is of interest in view of the natural ascension of lactobacilli from the gastro-intestinal to the vaginal tract.

As our understanding of lactobacilli, probiotics and the vaginal microbiota has evolved, it is becoming possible to explore the mechanisms that are key for successful applications to humans. Therefore, in the present study, we report the genome sequence of $L$. rhamnosus GR-1 and compare it to that of the gastro-intestinal probiotic L. rhamnosus strain GG (Kankainen et al., 2009), the model dairy isolate L. rhamnosus LC705 (Kankainen et al., 2009) as well as the other related L. rhamnosus strains currently in the NCBI database. This was done for two reasons: to investigate (i) inter-strain differences, and (ii) why L. rhamnosus GR-1 appears to be better adapted to the vagina, but less to the intestinal tract, than L. rhamnosus GG (Gardiner et al., 2002; Colodner et al., 2003). Insight into the genome of L. rhamnosus GR-1, and its comparison with related strains from other isolation sources, may help the identification of molecules playing roles in hostmicrobe and microbe-microbe interactions and the elucidation of the strain's functional and niche-adaptation mechanisms. This could particularly be of interest for approaches that aim at evading pathogens in the vaginal niche, as exemplified in our recent manuscript (Petrova et al., 2016). Furthermore, detailed knowledge about the genome of L. rhamnosus GR1 will provide a better understanding on the colonization and adaptation capacity of this strain following probiotic administration.

\section{MATERIALS AND METHODS}

\section{Bacterial Strains and Growth Conditions Used in This Study}

Lactobacilli were grown standard at $37^{\circ} \mathrm{C}$ in MRS medium (Difco) in non-shaking conditions. Modified de Man Rogosa Sharpe medium (mMRS), in which glucose was replaced by the same concentration of varied sugars $(20 \mathrm{~g} / \mathrm{L})$, was used as a growth medium to determine differences in the sugar utilization between L. rhamnosus GG, L. rhamnosus GR-1, and L. rhamnosus LC705. Each sugar compound was tested in three biological repetitions and each of the experiment was repeated at least three times.

\section{DNA Isolation}

Lactobacillus rhamnosus GR-1 was cultured in MRS agar (SigmaAldrich, Belgium), incubated at $37^{\circ} \mathrm{C}$ micro-aerophically for $24 \mathrm{~h}$, and DNA prepared using Epicentre MasterPure ${ }^{\mathrm{TM}}$ DNA Purification Kit and dsDNA quality checked with eppendorf UV Biophotometer (Oceanside, CA, United States).

\section{Sequencing and Annotation}

Purified DNA was sent to Illumina (Illumina, Inc., San Diego, CA, United States) for library preparation and sequencing. We received 35 bp paired-end reads which were used for assembly by Velvet 1.0.13 using a kmer of 27, expected coverage of 40, and a coverage cutoff of 20. Using the draft assembly, open reading frames (ORFs) were first predicted and then annotated with GeneMark v.4.21 and additionally predicted by Glimmer. The genomic assembly is available under the ENA Accession No. PRJEB24764.

\section{Pan-Genome Analysis}

All available $L$. rhamnosus genomic assemblies (NCBI; 04/04/2017) were downloaded and their quality was evaluated using QUAST v4.3 (Gurevich et al., 2013). This resulted in 116 different genomic assemblies that were downloaded, of which $100+1$ (L. rhamnosus GR-1) passed quality control. These analyses included visualization of different quality control parameters (such as GC content, total genome length, N50 and number of N's per 100.000 bases). Only genomes with a $\mathrm{N} 50>10 \mathrm{~kb}$ and a number of N's per 100.000 bases lower than 500 were kept. In addition, the completeness of all genomes was checked using CheckM (Parks et al., 2015) and only genomes with a completeness $>95 \%$ were kept. The assemblies were annotated using Prokka v1.12 (Seemann, 2014) and the pan genome was calculated with Orthofinder v1.1.18 (Emms and Kelly, 2015) using the $-\mathrm{M}$ msa option. For further analysis, a core orthogroup is defined as an orthogroup present in $>95 \%$ of the genomes. The phylogenetic tree was constructed with RAxML v8.2.9 (Stamatakis, 2014) using the alignment of all single copy core orthogroups on amino acid level, after addition of Lactobacillus casei subsp. casei ATCC 393 (GCA_000829055) as outgroup, PROTCATWAG as substitution matrix and the autoMRE option. The gene content was further analyzed in $\mathrm{R}$ and visualized using the $\mathrm{R}$ package UpsetR v1.3.3. Furthermore, the functional capacity was explored by mapping all representative sequences against the eggNOG (v4.5) Bacillus database (HuertaCepas et al., 2016) and calculating distance matrices using the $\mathrm{R}$ Package vegan v2.4.4 as described in Wuyts et al. (2017). Hereby, representative sequences represent the most abundant sequence in each orthogroup. Visualization was done using the $\mathrm{R}$ package ggplot2 v2.2.1 (Wickham, 2009).

\section{Adhesion Assay to CaCo-2 and VK2/E6E7 Cells}

In vitro adhesion assays using the CaCo-2 (ATCC HTB $37^{\mathrm{TM}}$ ) and VK2/E6E7 (ATCC CRL-2616 ${ }^{\mathrm{TM}}$ ) cell line were performed as previously described (Petrova et al., 2016). Briefly, differentiated Caco2 or VK2/E6E7 cells were washed two times with prewarmed 1x PBS and subsequently incubated for $1 \mathrm{~h}$ with $1.5 \mathrm{ml}$ of $10^{7}$ bacterial cells. After incubation at $37^{\circ} \mathrm{C}$ for $1 \mathrm{~h}$, the epithelial cell cultures were washed twice with pre-warmed PBS. Subsequently, $100 \mu \mathrm{l}$ of trypsin-EDTA (1x) (Invitrogen) was added to each well and incubated for $10 \mathrm{~min}$ at $37^{\circ} \mathrm{C}$. Finally, $900 \mu \mathrm{l}$ of PBS was added, mixed and serial dilutions were plated out. Plates were incubated at $37^{\circ} \mathrm{C}$ for $72 \mathrm{~h}$. The 
adhesion ratio (percentage) was calculated by comparing the number of adherent cells to the cell number of the added original bacterial suspension $\left(10^{7} \mathrm{CFU} / \mathrm{ml}\right)$. Adhesion of L. rhamnosus GG, L. rhamnosus GR-1 and L. rhamnosus LC705 was tested in triplicate in three independent experiments.

\section{spaCBA Presence}

The presence of spaA (NCBI Protein database Accession No. BAI40953), spaB (BAI40954) and spaC (BAI40955) genes was evaluated by performing a BLASTp (Camacho et al., 2009) search against a database containing all protein sequences of the above mentioned genomic assemblies. Only hits with a percentage identity and query coverage higher than $80 \%$ were retained.

\section{EPS Isolation and Monomer Characterization}

Extracellular polysaccharide (EPS) molecules were isolated and quantified as described previously (Lebeer et al., 2009). Briefly, total EPS was extracted from L. rhamnosus GG and L. rhamnosus GR-1 cells by mild sonication followed by ethanol precipitation and dialysis against water $[6,000-$ to $8,000-\mathrm{Da}$ dialysis membrane (Spectra/Por, VWR International)]. The total amount of carbohydrate was estimated by the phenolsulfuric acid method. The neutral sugar monomer composition of the isolated polysaccharides was determined according to the method of Englyst and Cummings (1984) by gas chromatography after hydrolysis and derivatization to alditol acetates. $\beta$-D-Allose was used as an internal standard and calibration samples (glucose, galactose, rhamnose, and mannose) containing the expected monosaccharides were included with each set of samples.

\section{Induction of Cytokine Gene Expression in VK2/E6E7 Epithelial Cells}

The cytokine expression was performed as previously described (Lebeer et al., 2012) Briefly, VK2/E6E7 cells growing in 12well tissue culture plates were deprived of FBS 1 day before the mRNA induction experiments. L. rhamnosus GG, L. rhamnosus
GR-1, and L. rhamnosus LC705 cells were grown overnight in MRS medium and subsequently centrifuged at 2,000 $\times g$ at $4^{\circ} \mathrm{C}$ for $10 \mathrm{~min}$. After washing with $1 \mathrm{x}$ PBS, cells were resuspended in DMEM without FBS and adjusted to a final concentration of $1 \times 10^{7} \mathrm{CFU} / \mathrm{ml}$. A $1.5-\mathrm{ml}$ volume of the L. rhamnosus GG or L. rhamnosus GR1 cell suspension was then added to the VK2/E6E7 epithelial cells for $1.5 \mathrm{~h}$. Afterward, the epithelial cells were rinsed twice with prewarmed 1x PBS and subsequently 0.2-ml volume of PBS was added to each of the wells. RNA was extracted from the VK2/E6E7 cells by using the high pure RNA isolation kit (Roche) following the manufacturer's protocol. The cytokine genes expression were measurement by quantitative reverse transcription-PCR (qRT-PCR) by using reverse transcription (SuperScript III first-strand synthesis system; Invitrogen), and real-time DNA amplification (TaqMan universal PCR master mix; Applied Biosystems). For RT-qPCR amplification, the StepOnePlus Real Time PCR system (Applied Biosystems, Lennik, Belgium) was used. All primers and probes were designed based on published sequences and chemically synthesized by Integrated DNA Technologies (IDT) (Belgium) (Table 1). Purified plasmid DNA specific for each targeted cytokine gene served as cDNA plasmid standards and was used to quantify the respective cytokine in the test samples. Peptidylprolyl cis-trans isomerase A (PPIA), served as the housekeeping gene.

\section{Stress Survival Assays in Simulated Gastro-Intestinal and Vaginal Conditions}

Stress survival tests were performed as previously described (Lebeer et al., 2011). Briefly, simulated gastric juice was prepared using $3.5 \mathrm{~g} / \mathrm{l}$ glucose, $2.05 \mathrm{~g} / \mathrm{l} \mathrm{NaCl}, 0.60 \mathrm{~g} / 1 \mathrm{KH}_{2} \mathrm{PO}_{4}, 0.11 \mathrm{~g} / \mathrm{l}$ $\mathrm{CaCl}_{2}$, and $0.37 \mathrm{~g} / \mathrm{l} \mathrm{KCl}$, adjusted to $\mathrm{pH} 2.0$ using $1.0 \mathrm{M}$ $\mathrm{HCl}$, and autoclaved at $121^{\circ} \mathrm{C}$ for $15 \mathrm{~min}$ (Corcoran et al., 2005). Subsequently, $0.05 \mathrm{~g} / \mathrm{l}$ porcine bile (Sigma-Aldrich), $0.1 \mathrm{~g} / \mathrm{l}$ lysozyme (Sigma-Aldrich), and $13.3 \mathrm{mg} / \mathrm{l}$ pepsin (Sigma-Aldrich) were added as stock solutions prior to analysis.

TABLE 1 | Primers and probes used in this study for cytokine mRNA measurements.

\begin{tabular}{|c|c|c|c|}
\hline Target mRNA & Sequence $\left(5^{\prime}-3^{\prime}\right)$ & Primer or probe & Reference \\
\hline & CTGACACATAAACCCTGGAAT AATTC & $\mathrm{RV}$ & \\
\hline \multirow[t]{3}{*}{ IL-8 } & TGGCAGCCTTCCTGATTTCT & $\mathrm{FW}$ & Bullens et al., 2006 \\
\hline & TTAGCACTCCTTGGCAAAACTG & RV & \\
\hline & CAGCTCTGTGTGAAGGT & TaqMan probe & \\
\hline & ССTCTGATGGCACCACCAG & $\mathrm{RV}$ & \\
\hline & TAGCCCATGTTGTAGCAAACCCTCAAGCT & TaqMan probe & \\
\hline \multirow[t]{3}{*}{ IL-6 } & GAGGATACCACTCCCAACAGACC & FW & Bullens et al., 2006 \\
\hline & AAGTGCATCATCGTTGTTCATACA & $\mathrm{RV}$ & \\
\hline & CAGAATTGCCATTGCACAACTCTITCTCA & TaqMan probe & \\
\hline
\end{tabular}

*FW, forward primer; RV, reverse primer; TaqMan probe, dually labeled with 5' 6-carboxyfluorescein and 3' 6-carboxytetramethylrhodamine; PPIA, peptidyl-prolyl cis-trans isomerase A; IL-8, interleukin-8; TNF, tumor necrosis factor. 
For the stress survival assays, $10^{8} \mathrm{CFU} / \mathrm{ml}$ (based on estimations via optical density at $600 \mathrm{~nm}$ ) cells were resuspended in the appropriate volume of either simulated gastric juice or $0.1 \% \mathrm{vol} / \mathrm{vol} \mathrm{H}_{2} \mathrm{O}_{2}$. Suspensions were incubated at $37^{\circ} \mathrm{C}$ for 90 min with constant stirring. The percentage of survivals was calculated by comparing the exact colony forming units (plate counts) before and after addition to simulated gastric juice or $0.1 \% \mathrm{H}_{2} \mathrm{O}_{2}$. Phosphate-buffered saline (PBS) at $\mathrm{pH} 7.4$ was used as baseline (negative control). Each strain and/or condition was tested threefold, and each experiment was performed at least in triplicate.

\section{RESULTS}

\section{Phylogenetic Relationships Between L. rhamnosus Strains}

The draft genome sequence of $L$. rhamnosus GR-1 was determined based on the assembly of 266 contigs, and found to have a genome size of $2.89 \mathrm{Mbp}$, a whole genome GC $\%$ of $46.48 \%$ and a total of 2,714 predicted genes. The pan genome of L. rhamnosus was calculated using the L. rhamnosus GR-1 assembly and 100 publicly available strains [10 complete genomes and $90+1$ (LGR1) draft genomes]. Only genomes with a sufficient high completeness were kept for the analysis to avoid problems with the absence of certain genes. In total, this pan genome contained 281,225 genes which were clustered into 4,526 orthogroups, defined as the group of genes descended from a single gene in the most recent common ancestor (Emms and Kelly, 2015). 2,143 orthogroups were found to be core orthogroup, while the remaining 2,383 were identified as accessory.

Next, all single-copy core orthogroups (952 in total) were used to construct a high resolution phylogenetic tree (Figure 1). Lactobacillus casei subsp. casei ATCC 393 (GCA_000829055) was chosen as outgroup, based on the most recent comparative genomics study (Wuyts et al., 2017). As shown in Figure 1, strain L. rhamnosus GR-1 shows high similarity with L. rhamnosus strain 51B (isolated from the vagina of a healthy Russian woman) and strain L. rhamnosus 906_LRHA (origin unclear) based on their 952 single copy core orthogroups. Similarly, strains L. rhamnosus Lc705, L. rhamnosus Lrh25, and L. rhamnosus Lrh26 are phylogenetically very closely related, as well as L. rhamnosus GG (ATCC 53103) and 17 other L. rhamnosus strains. Of note, in addition to L. rhamnosus GR-1 and L. rhamnosus $51 \mathrm{~B}$, two other genomic assemblies were annotated as vaginal isolates (strains BPL5 and Lhr31), which also seem to cluster together in a separate small clade. In contrast, isolates from all other isolation sources seem to be spread all over the phylogenetic tree.

\section{Phylogenetic Relationships Between Three Well-Known and Widely Used}

\section{L. rhamnosus Strains}

Subsequently, we focused in detail on the phylogenetic relationship between strains GR-1, GG and LC705, as these

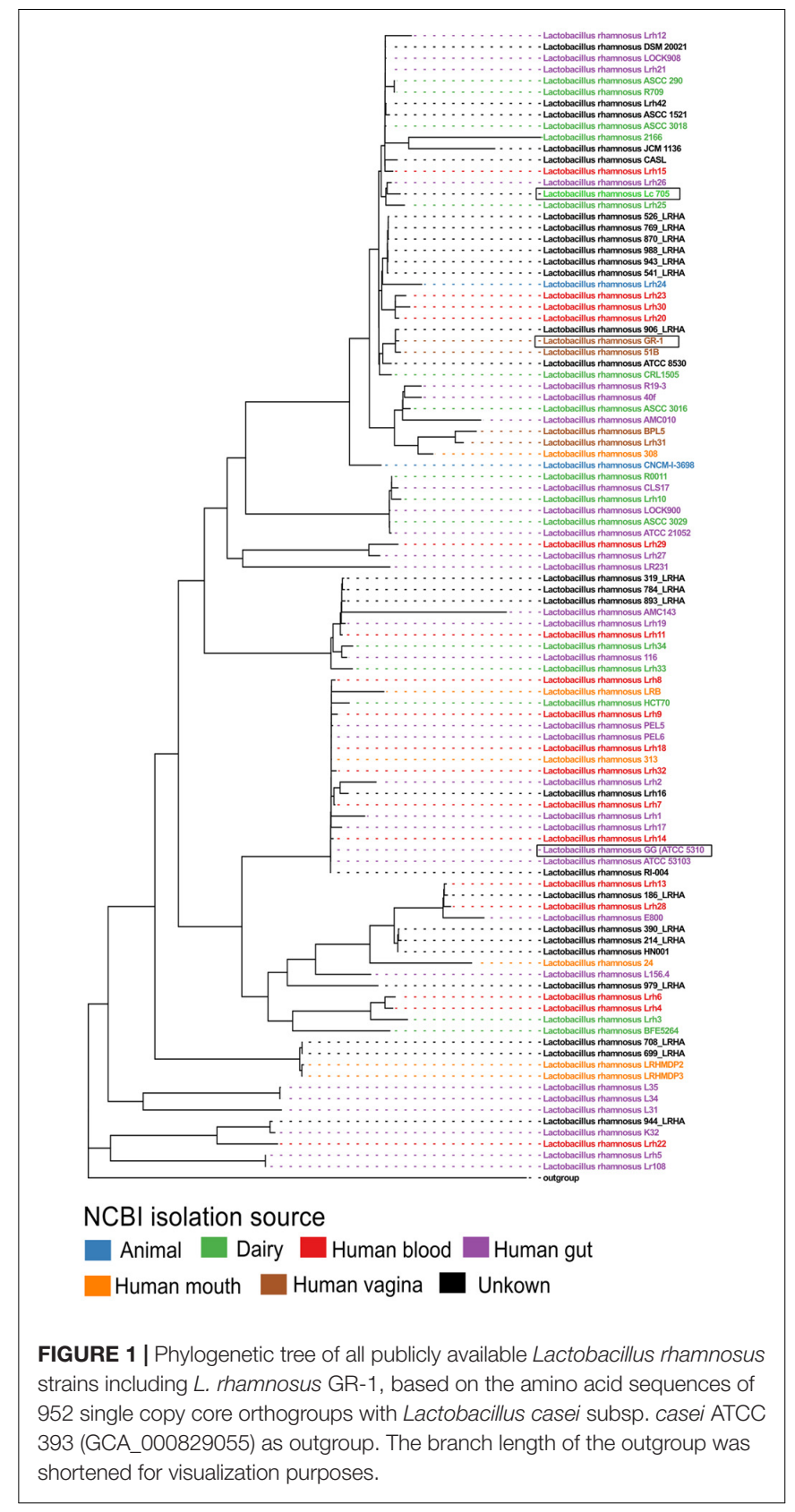

are widely studied and commercially available probiotics with different isolation source. The three strains appear to have 2,311 orthogroups in common (Figure 2A). L. rhamnosus GR-1 is missing 60 orthogroups between L. rhamnosus GG and L. rhamnosus LC705. Strains L. rhamnosus LC705 and L. rhamnosus GR-1 have 247 orthogroups in common which are not present in L. rhamnosus GG. Within this latter group, many metabolic genes related to carbohydrate metabolism and complex protein degradation are present. Strains L. rhamnosus GG and L. rhamnosus GR-1 have 39 orthogroups in common which are not present in L. rhamnosus LC705, suggesting that they are putative factors of relevance within the human body. Of most importance to our objectives, L. rhamnosus GR-1 has 48 unique 


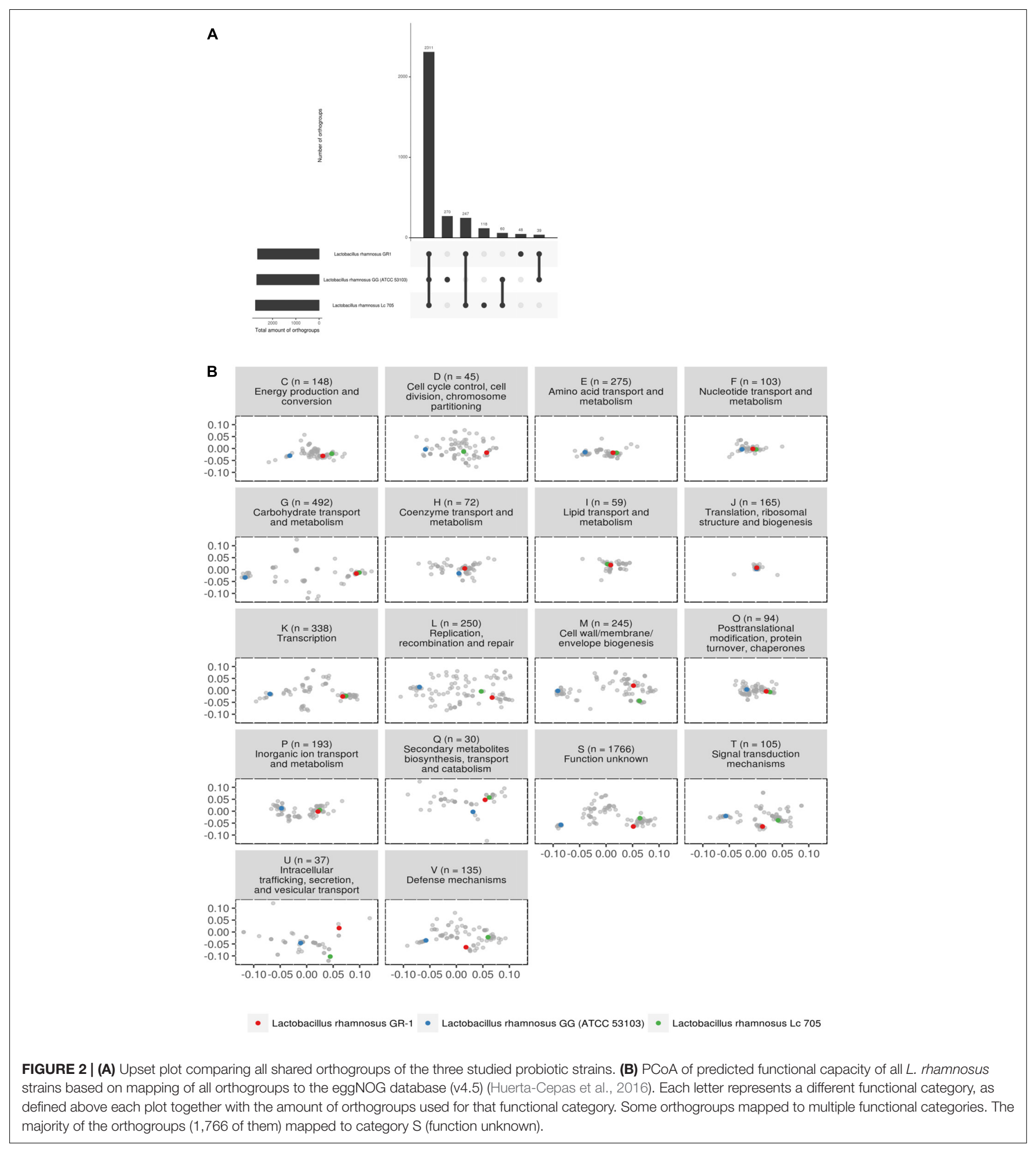

orthogroups not present in L. rhamnosus GG or L. rhamnosus L705, while L. rhamnosus GG has 270 unique orthogroups not present in L. rhamnosus GR-1 or LC705.

The predicted functional capacity of the three probiotic strains was further studied in relationship to all other L. rhamnosus genomes by mapping all orthogroups to the eggNOG database (v4.5) (Huerta-Cepas et al., 2016). Figure 2B shows that for most functional categories, the genomes clustered according to the phylogeny described in Figure 1. In general, the distance between strain GR-1 and LC705 is much smaller than the distance between GR-1 and strain GG. However, some categories show overlapping orthogroup composition, pointing toward functional 
conservation between $L$. rhamnosus GG, L. rhamnosus GR-1, and L. rhamnosus LC705. These include category F, representing nucleotide transport and metabolism; category $\mathrm{H}$ covering coenzyme transport and metabolism; category I representing lipid transport and metabolism; category J, covering translation, ribosomal structure and biogenesis function; and category $\mathrm{O}$, representing post-translational modification and chaperons. On the other hand, and of special interest, are the functional categories that show multiple clusters, suggesting a different functional capacity between these L. rhamnosus strains. For example, category $\mathrm{K}$ show that strain GR-1 and LC705 have a similar composition of orthogroups related to transcription, while strain GG seems to show a different composition. Likewise category G, representing orthogroups related to carbohydrate transport and metabolism, suggests a different carbohydrate usage between the vaginal and dairy strains (GR-1 and LC705) and strain GG.

\section{Carbohydrate Utilization}

Since large differences in carbohydrate metabolism-encoding genes were found between L. rhamnosus GG and L. rhamnosus GR-1, with the latter having most of its carbohydrate utilization capacity in common with the dairy strain L. rhamnosus LC705, we subsequently experimentally validated major differences in carbohydrate utilization between these strains (Figure 3A).

In contrast to L. rhamnosus GG, the L. rhamnosus GR-1 genome contains unique genes for lactose utilization, similar to L. rhamnosus LC705, and we could confirm experimentally that L. rhamnosus GR-1 can ferment lactose while L. rhamnosus GG cannot (Figure 3B). L. rhamnosus GR-1 appears to also more efficiently metabolize maltose (Figure 3C). In addition, L. rhamnosus GR-1 is able to metabolize rhamnose (Figure 3D). L. rhamnosus GR-1 also showed to have an enhanced capacity to metabolize ribose in agreement with LGR-1 having genes GR1_01559-GR1_01561 (Figure 3E). Furthermore, L. rhamnosus GR-1 shows a typical bi-phase growth curve when sorbitol is provided as main sugar source (Figure 3F). L. rhamnosus GR1, similar to L. rhamnosus LC705, was not able to grow on fucose, in comparison to L. rhamnosus GG which shows a slow but significant growth in the presence of this carbon source (Figure 3G). Finally, the growth of L. rhamnosus GR-1 and L. rhamnosus GG was examined in the presence of glycogen, which was previously reported to be an important sugar source in the vaginal niche. However, no growth was observed for both of the strains under the tested condition (data not shown).

\section{Adhesion and Presence of SpaCBA Pili}

The spaCBA-encoded pili that are the key adhesins for L. rhamnosus GG to bind to human intestinal mucus and intestinal epithelial cells (Kankainen et al., 2009; Lebeer et al., 2012), are absent from the L. rhamnosus GR-1 and L. rhamnosus LC705 genome sequence, as confirmed by PCR. This is also reflected by the phenotypic comparison, in which L. rhamnosus GR-1 and L. rhamnosus LC705 behave like the L. rhamnosus GG spaCBA gene deletion mutant lacking pili in terms of adhesion to intestinal epithelial CaCo-2 cells and VK/E6E7 vaginal epithelial cells (Figure 4A). In addition, mining other publicly available genome sequences of vaginal $L$. rhamnosus strains suggest that they all lack pili (Figure 4B). Of note, the presence of spaCBA genes is more prevalent in gastro-intestinal isolates than in dairy isolates. However, not all gastro-intestinal strains bear these spaCBA genes, confirming that pili form a strain-specific characteristic acquired during evolution.

\section{Extracellular Polysaccharide (EPS) Production and Immunomodulation}

Extracellular polysaccharide production is an important adaptation factor in lactobacilli. The galactose-rich rhamnosecontaining EPS molecules of L. rhamnosus GG form a protective shield against innate immune factors in the intestine including cathelicidins and complement factors (Lebeer et al., 2011). Therefore, a EPS cluster comparative analysis was performed for L. rhamnosus GG versus L. rhamnosus GR-1 (Figure 5A). Similarity can be observed for genes predicted to encode for the regulation of EPS production and polymerization, i.e., genes $w z d$ and wze located in the beginning of the EPS operon and $w z r$ and $w z b$ genes located in the end of the cluster. The EPS cluster of L. rhamnosus GR-1 appears to further contain unique glycosyltransferases for the steps of EPS biosynthesis (schematically represented on Figure 5B). The major differences between the two strains was the absence of NDP-sugar biosynthesis genes in the genome of L. rhamnosus GR-1 (represented with blue arrows in Figure 5A). The NDPsugar biosynthesis genes were predicted to be involved in the synthesis of galactose-rich rhamnose- containing EPS cluster in L. rhamnosus GG (Lebeer et al., 2009). To experimentally compare the neutral sugar monomer composition, cell bound EPS was isolated from L. rhamnosus GR-1 and L. rhamnosus GG. In contrast to the galactose-rich rhamnose-containing EPS molecules of L. rhamnosus GG (Lebeer et al., 2009), those of L. rhamnosus GR-1 are glucose-rich and completely lack rhamnose (Figure 6A), in agreement with the absence of $r m l A-D$ genes from its genome sequence (Figure 5A). We were able to experimentally show that $L$. rhamnosus GR-1 mediates different cytokine responses in vaginal VK2/E6E7 epithelial cells compared to L. rhamnosus GG (Figure 6B). While L. rhamnosus GG and L. rhamnosus LC705 induced the mRNA expression of the pro-inflammatory markers tumor necrosis factor (TNF) mRNA (twofold above background), L. rhamnosus GR-1 did not under the tested conditions. Further L. rhamnosus GG, but not L. rhamnosus LC705 and L. rhamnosus GR-1, was able to induce interleukin-8 (IL-8) with twofold above background. All tested strain induced IL-6 with twofold above background for L. rhamnosus LC705 and L. rhamnosus GR-1 and 3.5-fold for L. rhamnosus GG (Figure 6B).

\section{Stress Resistance in Simulated Gut and Vaginal Conditions}

Depending on the probiotic administration method, surviving gastric acidity is a key determinant for optimal survival for L. rhamnosus GG to reach the intestine and L. rhamnosus GR-1, taken orally to reach the rectum and vagina. In simulated gastric juice, L. rhamnosus GG was shown to survive significantly better 


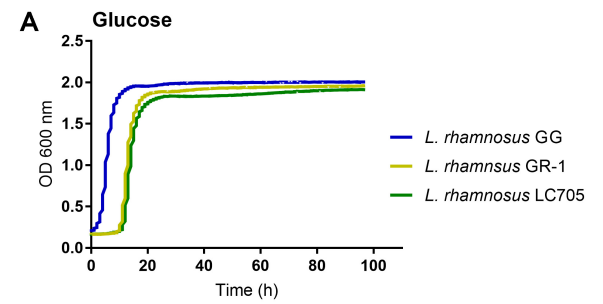

C

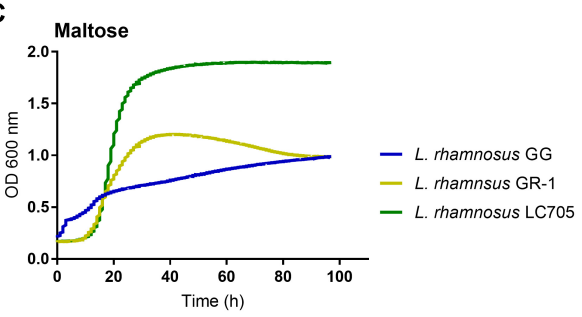

E

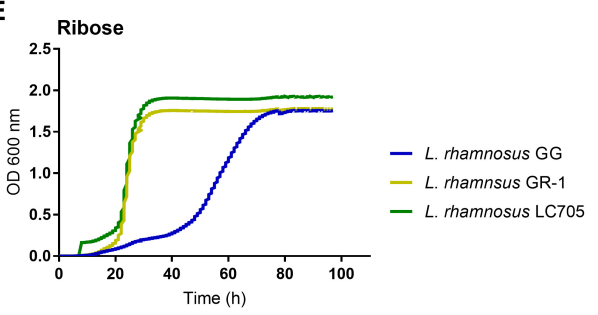

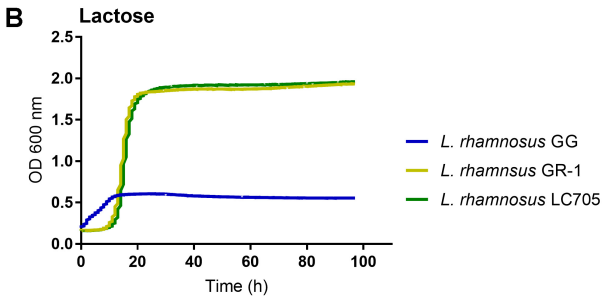

D
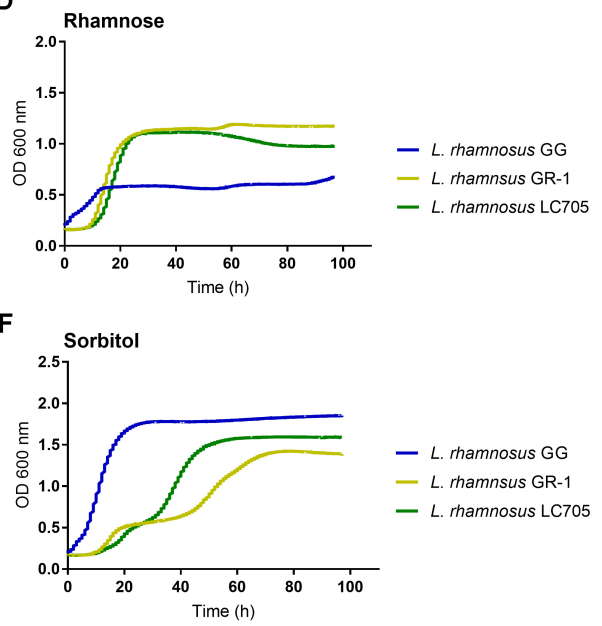

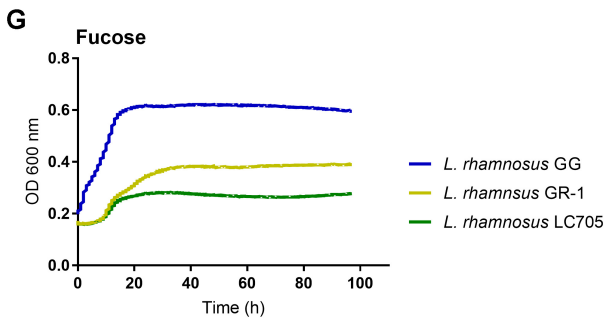

FIGURE 3 | Comparison of the metabolic capacity of L. rhamnosus GG, L. rhamnosus GR-1, and L. rhamnosus LC705. All the experiments were performed in modified MRS in which the main carbon source being glucose (A) was replaced with lactose (B), maltose (C), rhamnose (D), ribose (E), sorbitol (F), and fucose (G)

than L. rhamnosus GR-1 and L. rhamnosus LC705 (Figure 6C). On the other hand, L. rhamnosus GR-1 showed a better survival capacity under oxidative stress, particularly relevant in the vagina, as experimentally documented by an significantly enhanced resistance to $0.1 \% \mathrm{vol} / \mathrm{vol}$ hydrogen peroxide $\left(\mathrm{H}_{2} \mathrm{O}_{2}\right)$ compared to L. rhamnosus GG and L. rhamnosus LC705 (Figure 6C). This phenotype might be related to the presence of a putative thioredoxin genes (GR1_00460,GR1_01468, and GR1_01733) in L. rhamnosus GR-1.

\section{DISCUSSION}

While the genome sequences of various gastro-intestinal and dairy probiotic Lactobacillus strains have been published since 2004, including Lactobacillus acidophilus NCFM (Altermann et al., 2005), Lactobacillus salivarius subsp. salivarius UCC118 (Claesson et al., 2006) Lactobacillus gasseri ATCC 33323 (Azcarate-Peril et al., 2008) and L. rhamnosus GG (Kankainen et al., 2009), the urogenital probiotic strains are lagging behind.
Nevertheless, probiotic application of lactobacilli hold greater promise in the female vagina, because of the dominance of lactobacilli in these niches under health conditions, and their depletion upon disease (Petrova et al., 2015).

Here we report that L. rhamnosus GR-1 has a genome size of $2.91 \mathrm{Mbp}$, in agreement with other L. rhamnosus strains. For comparison, L. iners has only a genome size of only 1.3 Mbp (Macklaim et al., 2011) reflecting large reductive genome evolution (Frese et al., 2011) and a potential symbiotic lifestyle (Petrova et al., 2017). Even though we only know the source of isolation of these strains, the present genome comparison of L. rhamnosus GR-1, L. rhamnosus GG, and L. rhamnosus LC705 and the other L. rhamnosus genomes publicly available gives a snapshot of which predicted functional capacity correlates with the preferred niche of these strains.

Most apparent is the presence of pili-encoding genes for intestinal mucus adhesion only in L. rhamnosus GG, and not in L. rhamnosus GR-1 and L. rhamnosus LC705, in agreement with the fact that the energy-expensive biosynthesis of these long heteropolymeric structures only provides a competitive 


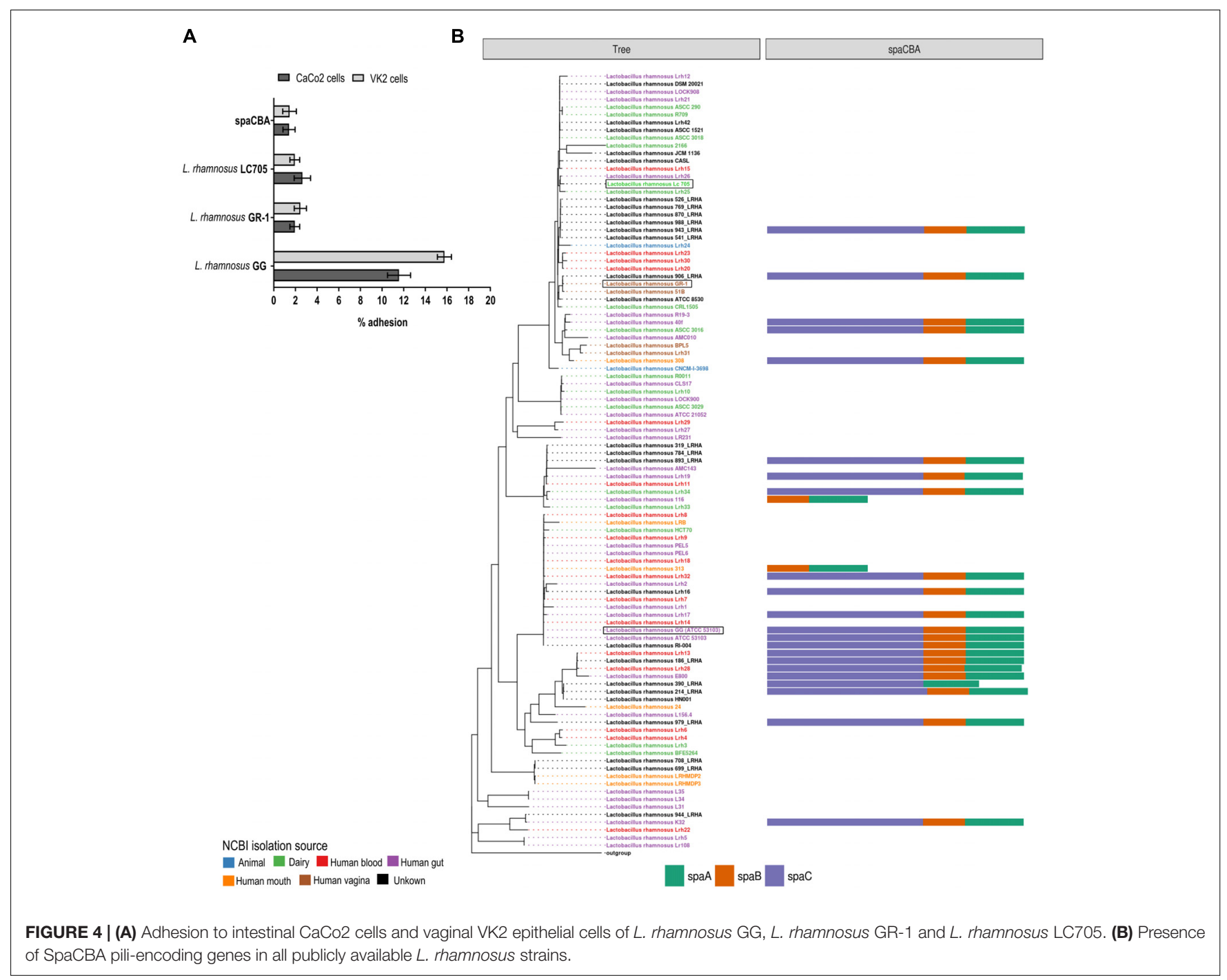

advantage in the gastro-intestinal tract. Perhaps the absence of pili and relatively low adhesion capacity of L. rhamnosus GR1 compared to $L$. rhamnosus GG might actually promote the natural ascension of this strain from the gastro-intestinal tract to the vagina via the rectum, since it was previously shown in vivo that L. rhamnosus GG is not able to colonize the human vagina as efficiently as L. rhamnosus GR-1 (Gardiner et al., 2002; Colodner et al., 2003). Our results are in agreement with the previous comparative genomic analysis of L. rhamnosus strains in which the spaCBA pili gene cluster was prevalent in human isolates, but rarely detected in dairy isolates (Douillard et al., 2013). Similarly, the authors observed that intestinal L. rhamnosus isolates were the most prevalent group encoding SpaCBA pili, but none of the vaginal and oral isolates produced pili (Douillard et al., 2013).

On the other hand, the L. rhamnosus GR-1 genome seems to encode a number of traits suited to vaginal persistence, unlike the gut targeted probiotic L. rhamnosus GG. Of note, the genome and functional analysis of L. rhamnosus GR-1 reflects a more versatile carbohydrate utilization capacity than L. rhamnosus GG. Strain L. rhamnosus GR-1 was indeed predicted and experimentally shown here to utilize maltose, lactose, ribose and rhamnose, carbohydrates, which were previously shown to be expressed by human vaginal tissue (Gregoire, 1963; Wang et al., 2015) and can therefore be used an important carbon source for vaginal species. Interestingly, these carbohydrates are also highly relevant for dairy applications and were preferable carbon source for L. rhamnosus LC705. In contrast, we observed that L. rhamnosus GG utilize fucose better compared to L. rhamnosus GR-1 and L. rhamnosus LC705, as also reported by other authors (Douillard et al., 2013). This highlights the importance of fucose as carbon source in the intestinal tract, where fucosylated compounds such as human mucin and other glycoproteins are highly abundant.

In addition, we were able to show that L. rhamnosus GR-1 can withstand much better oxidative stress in comparison with L. rhamnosus GG. This might be important for better survival and adaptation in the vaginal niche. Notably, L. rhamnosus GG survived better in simulated gastric juice than L. rhamnosus GR-1. This low survival capacity of L. rhamnosus GR-1 might affect its capacity to reach the intestine, rectum, and vagina to help prevent or treat vaginally associated disorders, such 

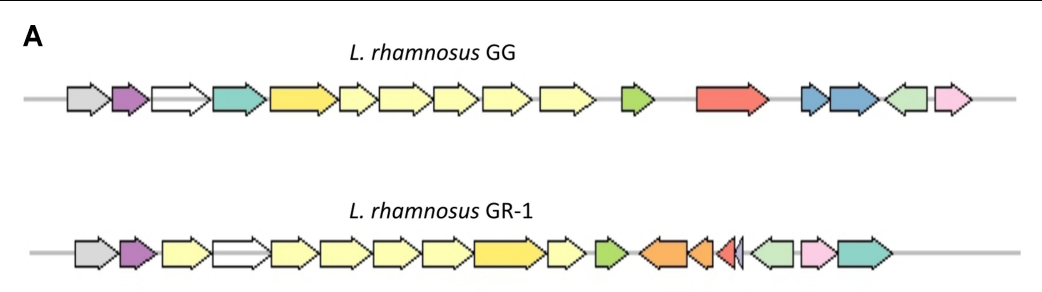

\section{B}

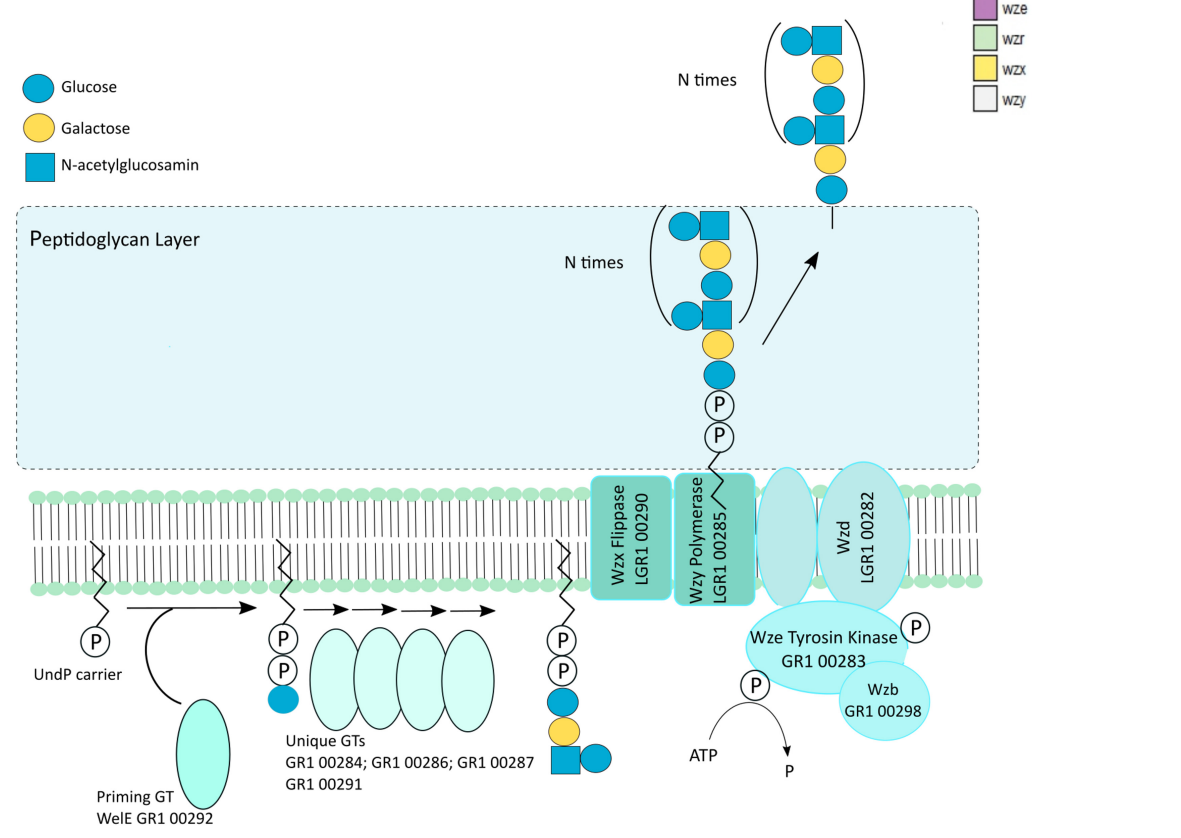

FIGURE 5 | (A) Organization of the EPS gene cluster of L. rhamnosus GR-1 in comparison with the EPS gene cluster organization of $L$. rhamnosus GG. (B) Schematic representation of the putative steps in EPS biosynthesis by L. rhamnosus GR-1 that are encoded within the EPS gene cluster.

as bacterial vaginosis, aerobic vaginitis and yeast infections. Therefore, applying L. rhamnosus GR-1 vaginally, as capsules, might be a better choice for the treatment of vaginal disorders and infections. This will result in a local administration of the strain and will ensure its better survival and colonization capacity, probably also resulting in a better treatment outcome. Yet, orally administrated tablets or capsules are better accepted by patients and still show an improvement of vaginal diseases. Hereto, they are often given twice daily at concentration of at least $10^{9} \mathrm{CFU}$, which may guarantee the survival and the passage of sufficient numbers of L. rhamnosus GR-1 to the vaginal niche. Of note, vaginally administrated capsules are often applied only once a day which may also lower the cost of the treatment. Nevertheless, future studies should focus on the effect of orally vs. vaginally applied L. rhamnosus GR-1 to confirm which administration route is more effective.

Another striking difference is that the L. rhamnosus GR1 genome encodes at least one EPS-associated cluster, and was shown to produce glucose-rich EPS molecules that lack rhamnose. The EPS gene cluster of L. rhamnosus GG (LGG_02036-LGG_02054), encoding galactose-rich EPS molecules, is important in survival in the gastro-intestinal tract, by protecting against innate immune factors (Lebeer et al., 2011).
Our present finding suggests there could be different recognition of the two L. rhamnosus strains by immune cells in the gut and in the vagina. The Type I mucosal surface in the gut has a simple columnar epithelium, while the type II mucosal surface in the vagina and ectocervix have a protective squamous epithelial layer lacking IgA transport mechanisms. In addition, while the submucosa of the Type I surface is rich in mucosa-associated lymphoid tissue, the Type II mucosal surface has specialized highly phagocytic Langerhans cells in the epithelium, yet only contains a sparse network of dendritic cells, macrophages and rare lymphocytes in the submucosa (Iwasaki, 2010). We showed here that L. rhamnosus GR-1 did not induce expression of TNF and IL- 8 by VK2/E6E7, while L. rhamnosus GG was able to induce expression of TNF, IL- 8 and IL- 6 under the tested conditions, indicating a different recognition of the two strains by epithelial cells.

Interesting, Douillard et al. (2013) reported that L. rhamnosus species can be separated in two main geno-phenotypes called A and $\mathrm{B}$, which correspond to adaptation to different niches. The strains belonging to the geno-phenotype A were characterized by a lack of SpaCBA pili and the capacity to utilize lactose, maltose, and rhamnose. According to our results, L. rhamnosus GR-1 can be classified within A geno-phenotype. The authors observed 


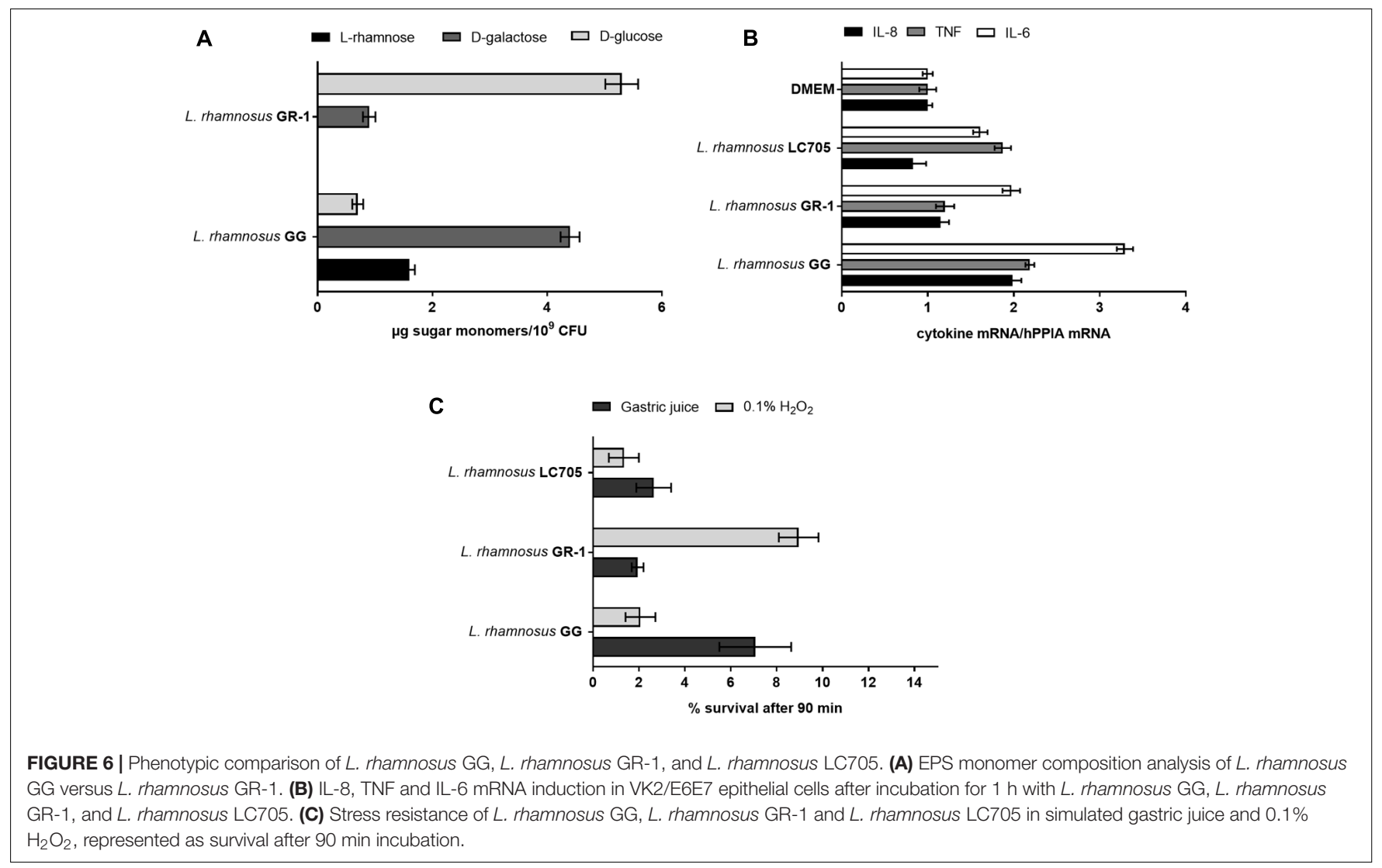

that other vaginal and dairy strains can also be classified as A geno-phenotype or as strains adapted to more stable nutrientrich environment, such as the vaginal niche. These strains appear to have lost various biological functions such as antimicrobial activity, stress resistance, adaptability and fitness to a range of habitats, all of which may not be required in the vaginal niche where lower bacterial diversity is observed. The geno-phenotype B, the one in which L. rhamnosus GG is classified, depicts strains adapted to the intestinal niche, characterized by production of pili, bile resistance and utilization L-fucose. These strains show adaptation to more diverse environments with constant changes in nutrition source, bacterial density, and host effects.

In summary, whilst L. rhamnosus is not a dominant species in the vagina of healthy women (Zhang et al., 2012), the selection of L. rhamnosus GR-1 as a probiotic, originally because of antagonism to uropathogens, is supported by the finding of a number of characteristics well-suited to vaginal persistence (Cadieux et al., 2002) and clinical effectiveness (Gardiner et al., 2002; Irvine et al., 2010). By identifying probiotic strain capabilities, it will 1 day be possible to improve the alignment of the best strain suited for a particular aberration in the vaginal niche.

\section{AUTHOR CONTRIBUTIONS}

MP, SL, and GR designed the experiments and wrote the manuscript. SW performed the genome comparison analysis and wrote part of the manuscript. JM, GG, and GR sequenced and annotated the genome of L. rhamnosus GR-1. MP and TV performed the experimental work. All the authors reviewed the manuscript and included additional suggestions and corrections.

\section{FUNDING}

MP was supported with a previous post-doctoral grant from the Fund for Scientific Research (FWO Vlaanderen). SL was supported from the FWO Vlaanderen with a previous postdoctoral grant, a research grant KaN 28960 and IWT-SBO funding (IWT1500054). SW holds an IWT-SB doctoral grant (SB 141198). Work at KU Leuven was supported by BOF program financing from the KU Leuven.

\section{ACKNOWLEDGMENTS}

We acknowledge Geert Schoofs, Camille Allousius, Jeroen Snelders, and Christophe Courtin for the EPS isolation and monomer analysis and Dr. Wayne Miller and Marc Monachese for assistance with EPS GC/MS. We also thank Stijn Wittouck for his valuable input regarding data analysis.

Some computational resources and services used in this work were provided by the VSC (Flemish Supercomputer Center), funded by the Research Foundation-Flanders (FWO) and the Flemish Government-Department EWI. 


\section{REFERENCES}

Altermann, E., Russell, W. M., Azcarate-Peril, M. A., Barrangou, R., Buck, B. L., McAuliffe, O., et al. (2005). Complete genome sequence of the probiotic lactic acid bacterium Lactobacillus acidophilus NCFM. Proc. Natl. Acad. Sci. U.S.A. 102, 3906-3912. doi: 10.1073/pnas.0409188102

Azcarate-Peril, M. A., Altermann, E., Goh, Y. J., Tallon, R., Sanozky-Dawes, R. B., Pfeiler, E. A., et al. (2008). Analysis of the genome sequence of Lactobacillus gasseri ATCC 33323 reveals the molecular basis of an autochthonous intestinal organism. Appl. Environ. Microbiol. 74, 4610-4625. doi: 10.1128/AEM.000 54-08

Bruce, A. W., Chadwick, P., Hassan, A., and VanCott, G. F. (1973). Recurrent urethritis in women. Can. Med. Assoc. J. 108, 973-976.

Bruce, A. W., and Reid, G. (1988). Intravaginal instillation of lactobacilli for prevention of recurrent urinary tract infections. Can. J. Microbiol. 34, 339-343. doi: $10.1139 / \mathrm{m} 88-062$

Bullens, D. M., Truyen, E., Coteur, L., Dilissen, E., Hellings, P. W., Dupont, L. J., et al. (2006). IL-17 mRNA in sputum of asthmatic patients: linking $\mathrm{T}$ cell driven inflammation and granulocytic influx. Respir. Res. 7:135. doi: 10.1186/14659921-7- 135

Cadieux, P., Burton, J., Gardiner, G., Braunstein, I., Bruce, A. W., Kang, C. Y., et al. (2002). Lactobacillus strains and vaginal ecology. JAMA 287, 1940-1941. doi: 10.1001/jama.287.15.1940

Camacho, C., Coulouris, G., Avagyan, V., Ma, N., Papadopoulos, J., Bealer, K., et al. (2009). BLAST+: architecture and applications. BMC Bioinformatics 10:421. doi: 10.1186/1471-2105-10-421

Chan, R. C., Reid, G., Irvin, R. T., Bruce, A. W., and Costerton, J. W. (1985). Competitive exclusion of uropathogens from human uroepithelial cells by Lactobacillus whole cells and cell wall fragments. Infect. Immun. 47, 84-89.

Claesson, M. J., Li, Y., Leahy, S., Canchaya, C., van Pijkeren, J. P., CerdenoTarraga, A. M., et al. (2006). Multireplicon genome architecture of Lactobacillus salivarius. Proc. Natl. Acad. Sci. U.S.A. 103, 6718-6723. doi: 10.1073/pnas. 0511060103

Colodner, R., Edelstein, H., Chazan, B., and Raz, R. (2003). Vaginal colonization by orally administered Lactobacillus rhamnosus GG. Isr. Med. Assoc. J. 5, 767-769.

Corcoran, B. M., Stanton, C., Fitzgerald, G. F., and Ross, R. P. (2005). Survival of probiotic lactobacilli in acidic environments is enhanced in the presence of metabolizable sugars. Appl. Environ. Microbiol. 71, 3060-3067. doi: 10.1128/ AEM.71.6.3060-3067.2005

Douillard, F. P., Ribbera, A., Kant, R., Pietila, T. E., Jarvinen, H. M., Messing, M., et al. (2013). Comparative genomic and functional analysis of 100 Lactobacillus rhamnosus strains and their comparison with strain GG. PLoS Genet. 9:e1003683. doi: 10.1371/journal.pgen.1003683

Emms, D. M., and Kelly, S. (2015). OrthoFinder: solving fundamental biases in whole genome comparisons dramatically improves orthogroup inference accuracy. Genome Biol. 16:157. doi: 10.1186/s13059-015-0 $721-2$

Englyst, H. N., and Cummings, J. H. (1984). Simplified method for the measurement of total non-starch polysaccharides by gas-liquid chromatography of constituent sugars as alditol acetates. Analyst 109, 937-942. doi: 10.1039/AN9840900937

Frese, S. A., Benson, A. K., Tannock, G. W., Loach, D. M., Kim, J., Zhang, M., et al. (2011). The evolution of host specialization in the vertebrate gut symbiont Lactobacillus reuteri. PLoS Genet. 7:e1001314. doi: 10.1371/journal. pgen. 1001314

Gardiner, G. E., Heinemann, C., Bruce, A. W., Beuerman, D., and Reid, G. (2002). Persistence of Lactobacillus fermentum RC-14 and Lactobacillus rhamnosus GR-1 but not L. rhamnosus GG in the human vagina as demonstrated by randomly amplified polymorphic DNA. Clin. Diagn. Lab. Immunol. 9, 92-96. doi: 10.1128/CDLI.9.1.92-96.2002

Giulietti, A., Overbergh, L., Valckx, D., Decallonne, B., Bouillon, R., and Mathieu, C. (2001). An overview of real-time quantitative PCR: applications to quantify cytokine gene expression. Methods 25, 386-401. doi: 10.1006/meth. 2001.1261

Gregoire, A. T. (1963). Carbohydrates of human vaginal tissue. Nature 198:996. doi: $10.1038 / 198996 \mathrm{a} 0$
Gurevich, A., Saveliev, V., Vyahhi, N., and Tesler, G. (2013). QUAST: quality assessment tool for genome assemblies. Bioinformatics 29, 1072-1075. doi: 10 . 1093/bioinformatics/btt086

Hayeshi, R., Hilgendorf, C., Artursson, P., Augustijns, P., Brodin, B., Dehertogh, P., et al. (2008). Comparison of drug transporter gene expression and functionality in Caco-2 cells from 10 different laboratories. Eur. J. Pharm. Sci. 35, 383-396. doi: 10.1016/j.ejps.2008.08.004

Huerta-Cepas, J., Szklarczyk, D., Forslund, K., Cook, H., Heller, D., Walter, M. C., et al. (2016). eggNOG 4.5: a hierarchical orthology framework with improved functional annotations for eukaryotic, prokaryotic and viral sequences. Nucleic Acids Res. 44, D286-D293. doi: 10.1093/nar/gkv 1248

Irvine, S. L., Hummelen, R., Hekmat, S., Looman, C. W., Habbema, J. D., and Reid, G. (2010). Probiotic yogurt consumption is associated with an increase of CD4 count among people living with HIV/AIDS. J. Clin. Gastroenterol. 44, e201-e205. doi: 10.1097/MCG.0b013e3181d8fba8

Iwasaki, A. (2010). Antiviral immune responses in the genital tract: clues for vaccines. Nat. Rev. Immunol. 10, 699-711. doi: 10.1038/nri2836

Kankainen, M., Paulin, L., Tynkkynen, S., von, O. I, Reunanen, J., Partanen, P., et al. (2009). Comparative genomic analysis of Lactobacillus rhamnosus GG reveals pili containing a human- mucus binding protein. Proc. Natl. Acad. Sci. U.S.A. 106, 17193-17198. doi: 10.1073/pnas.0908876106

Kirjavainen, P. V., Laine, R. M., Carter, D. E., Hammond, J., and Reid, G. (2008). Expression of anti-microbial defense factors in vaginal mucosa following exposure to Lactobacillus rhamnosus GR-1. Int. J. Probiotics Prebiotics 3, 99-106.

Lebeer, S., Claes, I., Tytgat, H. L., Verhoeven, T. L., Marien, E., von, O., et al. (2012). Functional analysis of Lactobacillus rhamnosus GG pili in relation to adhesion and immunomodulatory interactions with intestinal epithelial cells. Appl. Environ. Microbiol. 78, 185-193. doi: 10.1128/AEM.06192-11

Lebeer, S., Claes, I. J., Verhoeven, T. L., Vanderleyden, J., and De Keersmaecker, S. C. (2011). Exopolysaccharides of Lactobacillus rhamnosus GG form a protective shield against innate immune factors in the intestine. Microb. Biotechnol. 4, 368-374. doi: 10.1111/j.1751-7915.2010.00199.x

Lebeer, S., Verhoeven, T. L., Francius, G., Schoofs, G., Lambrichts, I., Dufrene, Y., et al. (2009). Identification of a gene cluster for the biosynthesis of a long, galactose-rich exopolysaccharide in Lactobacillus rhamnosus GG and functional analysis of the priming glycosyltransferase. Appl. Environ. Microbiol. 75, 35543563. doi: 10.1128/AEM.02919-08

Macklaim, J. M., Gloor, G. B., Anukam, K. C., Cribby, S., and Reid, G. (2011). At the crossroads of vaginal health and disease, the genome sequence of Lactobacillus iners AB-1. Proc. Natl. Acad. Sci. U.S.A. 108(Suppl. 1), 4688-4695. doi: 10.1073/ pnas. 1000086107

McMillan, A., Dell, M., Zellar, M. P., Cribby, S., Martz, S., Hong, E., et al. (2011). Disruption of urogenital biofilms by lactobacilli. Colloids Surf B Biointerfaces 86, 58-64. doi: 10.1016/j.colsurfb.2011.03.016

Parks, D. H., Imelfort, M., Skennerton, C. T., Hugenholtz, P., and Tyson, G. W. (2015). CheckM: assessing the quality of microbial genomes recovered from isolates, single cells, and metagenomes. Genome Res. 25, 1043-1055. doi: 10. 1101/gr.186072.114

Petrova, M. I., Lievens, E., Malik, S., Imholz, N., and Lebeer, S. (2015). Lactobacillus species as biomarkers and agents that can promote various aspects of vaginal health. Front. Physiol. 6:81. doi: 10.3389/fphys.2015.00081

Petrova, M. I., Lievens, E., Verhoeven, T. L., Macklaim, J. M., Gloor, G., Schols, D., et al. (2016). The lectin-like protein 1 in Lactobacillus rhamnosus GR-1 mediates tissue-specific adherence to vaginal epithelium and inhibits urogenital pathogens. Sci. Rep. 6:37437. doi: 10.1038/srep37437

Petrova, M. I., Reid, G., Vaneechoutte, M., and Lebeer, S. (2017). Lactobacillus iners: friend or Foe? Trends Microbiol. 25, 182-191. doi: 10.1016/j.tim.2016.11. 007

Reid, G., Chan, R. C., Bruce, A. W., and Costerton, J. W. (1985). Prevention of urinary tract infection in rats with an indigenous Lactobacillus casei strain. Infect. Immun. 49, 320-324.

Reid, G., Charbonneau, D., Erb, J., Kochanowski, B., Beuerman, D., Poehner, R., et al. (2003). Oral use of Lactobacillus rhamnosus GR-1 and L. fermentum RC14 significantly alters vaginal flora: randomized, placebo-controlled trial in 64 healthy women. FEMS Immunol. Med. Microbiol. 35, 131-134. doi: 10.1016/ S0928-8244(02)00465-0 
Seemann, T. (2014). Prokka: rapid prokaryotic genome annotation. Bioinformatics 30, 2068-2069. doi: 10.1093/bioinformatics/btu153

Stamatakis, A. (2014). RAxML version 8: a tool for phylogenetic analysis and post-analysis of large phylogenies. Bioinformatics 30, 1312-1313. doi: 10.1093/ bioinformatics/btu033

Wang, L., Koppolu, S., Chappell, C., Moncla, B. J., Hillier, S. L., and Mahal, L. K. (2015). Studying the effects of reproductive hormones and bacterial vaginosis on the glycome of lavage samples from the cervicovaginal cavity. PLoS One 10:e0127021. doi: 10.1371/journal.pone.0127021

Wickham, H. (2009). ggplot2: Elegant Graphics for Data Analysis. New York, NY: Springer Verlag. doi: 10.1007/978-0-387-98141-3

Wuyts, S., Wittouck, S., De, B. I, Allonsius, C. N., Pasolli, E., Segata, N., et al. (2017). Large-scale pylogenomics of the Lactobacillus casei group highlights taxonomic inconsistencies and reveals novel cladeassociated features. mSystems 2:e0061-17. doi: 10.1128/mSystems.000 $61-17$
Zhang, R., Daroczy, K., Xiao, B., Yu, L., Chen, R., and Liao, Q. (2012). Qualitative and semiquantitative analysis of Lactobacillus species in the vaginas of healthy fertile and postmenopausal Chinese women. J. Med. Microbiol. 61, 729-739. doi: 10.1099/jmm.0.038687-0

Conflict of Interest Statement: The authors declare that the research was conducted in the absence of any commercial or financial relationships that could be construed as a potential conflict of interest.

Copyright (C) 2018 Petrova, Macklaim, Wuyts, Verhoeven, Vanderleyden, Gloor, Lebeer and Reid. This is an open-access article distributed under the terms of the Creative Commons Attribution License (CC BY). The use, distribution or reproduction in other forums is permitted, provided the original author(s) and the copyright owner are credited and that the original publication in this journal is cited, in accordance with accepted academic practice. No use, distribution or reproduction is permitted which does not comply with these terms. 